\title{
Two maps on affine type $A$ crystals and Hecke algebras
}

\author{
Nicolas Jacon* \\ Université de Reims Champagne-Ardennes \\ Laboratoire de Mathématiques UMR CNRS 9008 \\ Reims, France \\ nicolas.jacon@univ-reims.fr
}

Submitted: Feb 15, 2021; Accepted: May 5, 2021; Published: May 21, 2021

(C) N.Jacon. Released under the CC BY-ND license (International 4.0).

\begin{abstract}
We use the crystal isomorphisms of the Fock space to describe two maps on partitions and multipartitions which naturally appear in the crystal basis theory for quantum groups in affine type $A$ and in the representation theory of Hecke algebras of type $G(l, l, n)$.
\end{abstract}

Mathematics Subject Classifications: 20C08,05E10,17B37

\section{Introduction}

One of the first problems in the study of the representation theory of a finite group is to find a classification of its simple modules. The difficulty of this problem depends widely on the characteristic of the field of the representations. In the case of the symmetric group $\mathfrak{S}_{n}$ and in characteristic 0, it is known from the works of Frobenius in the beginning of the twentieth century that the irreducible representations are naturally labelled by the partitions of $n$. As a consequence, several fundamental questions around this theory are closely related to the combinatorics of partitions. For example, the dimensions of an irreducible representation is given by the number of standard tableaux with shape the diagram of the partition.

In positive characteristic $p>0$, the difficulty comes from the fact that the representation theory can be non semisimple. In this case, we know how to classify the irreducible representations: they are naturally labeled by the set of $p$-regular partitions but their structures are far from being completely understood (for example we do not even know

${ }^{*}$ Supported by ANR AHA JCJC ANR-18-CE40-0001. 
what are their dimensions). One way to attack this problem is to attach to the symmetric group, its Iwahori-Hecke algebra, which may be seen as a deformation of the group algebra. The representation theory of this algebra is indeed closely connected with the representation theory of the symmetric group, in particular in positive characteristic, and also to various other algebraic objects, such as the finite reductive groups, Cherednik algebras etc (see [2].)

The symmetric groups are particular cases of finite Coxeter groups, which are themselves particular cases of complex reflection groups. A complex reflection group is a finite group of complex matrices generated by "pseudo-reflections". Their classification is due to Shephard and Todd. If $W$ is an irreducible complex reflection group then either it belongs to an infinite series which is denoted by $G(l, p, n)$ and which depends on three parameters or it is in a list of 34 exceptional groups. Important works in the last decades have suggested that theses groups play a crucial role in Representation theory. In this paper, we will consider the complex reflection groups of type $G(l, 1, n)$, sometimes called "generalized symmetric groups", and $G(l, l, n)$. The aim is to study the analogues of the $p$-regular partitions for these algebras and the relations between them. In positive characteristic, since the works of Lascoux, Leclerc, Thibon and Ariki in the 90's, it is known that the representation theory of the Hecke algebras of these groups is closely related to the crystal basis theory for quantum groups. In particular, the construction of a remarkable combinatorial graph (the crystal graph in affine type $A$ ) leads to a classification of the simple modules of the Hecke algebra of type $G(l, 1, n)$ (also known as Ariki-Koike algebra) in the modular case by certain combinatorial objects called Uglov $l$-partitions. These classes of $l$-tuples of partitions may be seen as generalizations of the $p$-regular partitions (they coincide when $l=1$ ). These algebras include the cases of Hecke algebras of type $A$ - that is of the symmetric group - and $B$ - that is of the hyperoctohedral group (see $[1,2])$.

A lot of information on the representation theory of Hecke algebras of type $D$ (which is a deformation of the Weyl group of type $D$ ) or, more generally, of type $G(l, l, n)$ can be obtained from the $G(l, 1, n)$ case. In fact, these latter algebras can be seen as subalgebras of Hecke algebras of type $G(l, 1, n)$ and, by using a classical technics in group theory, namely the Clifford Theory, it is possible to produce all the simple modules by studying the restriction of the simple modules of the Hecke algebras of type $G(l, 1, n)$. This problem has been studied in various papers using different approaches (see $[4,5,3]$ and the references therein). The one developed in [4] and [6] in particular involves the existence of two combinatorial maps which are defined using the crystal graphs that we have already mentioned:

- The first map associates to each Uglov l-partition labelling a vertex of the crystal graph, another Uglov l-partition.

- The second one associates to each e-regular partition a certain Uglov l-partition.

The existence of such maps is non trivial and based on the structure of the associated crystal graphs. Moreover, their descriptions are only recursive on the size of the partitions/multipartitions involved. The relevance of these maps is that they allow to describe 
the restrictions of the simple modules of the Hecke algebras of type $G(l, 1, n)$ to the Hecke algebras of type $G(l, l, n)$.

The aim of this note is to recover, generalize and make explicit these results. The main tools of the proof are the crystal isomorphisms defined and described in [9]. Using them, the proofs become purely combinatorial and quite elementary. They also permits to explain how the approaches developed in [4] and in [3] are related.

aim of this note is to recover, generalize and make explicit these results. The main tools of the proof are the crystal isomorphisms defined and described in [9]. Using them, the proofs become purely combinatorial and quite elementary. They also permits to explain how the approaches developed in [4] and in [3] are related.

\section{Crystals}

In this part, we quickly recall some basic combinatorial notions, then we focus on the definition and on important properties of the crystals for Fock spaces. In all this section, we set $l \in \mathbb{Z}_{>0}$ and $e \in \mathbb{Z}_{>1}$.

\subsection{Generalities on Fock spaces and crystals}

A partition is by definition a nonincreasing sequence $\lambda=\left(\lambda_{1}, \ldots, \lambda_{m}\right)$ of nonnegative integers. If $\sum_{1 \leqslant i \leqslant m} \lambda_{i}=n$, we say that $\lambda$ is a partition of $n$. For $j=1, \ldots, l$, let $\lambda^{j}$ be a partition of $n_{j} \in \mathbb{Z}_{\geqslant 0}$ then we say that the $l$-tuple $\boldsymbol{\lambda}:=\left(\lambda^{1}, \ldots, \lambda^{l}\right)$ is an l-partition of $n$ if $\sum_{1 \leqslant j \leqslant l} n_{j}=n$. We denote by $\Pi^{l}(n)$ the set of $l$-partitions of rank $n$. The empty $l$-partition is by definition the unique partition of 0 and it is denoted by $\emptyset:=(\emptyset, \ldots, \emptyset)$. When $l=1$, the 1-partitions are identified with the partitions in an obvious way.

Let $\mathbf{s}=\left(s_{1}, \ldots, s_{l}\right) \in \mathbb{Z}^{l}$ (we say that $\mathbf{s}$ is a multicharge). Let $q$ be an indeterminate. The $\mathbb{Q}(q)$-vector space generated by all the $l$-partitions:

$$
\mathcal{F}_{q}:=\bigoplus_{n \in \mathbb{Z}_{\geqslant 0}} \bigoplus_{\lambda \in \Pi^{l}(n)} \mathbb{Q}(q) \boldsymbol{\lambda}
$$

is called the Fock space. Let $\mathcal{U}_{q}\left(\widehat{\mathfrak{s l}}_{e}\right)$ be the quantum group of affine type $A_{e-1}^{(1)}$. This is an associative $\mathbb{Q}(q)$-algebra with generators $e_{i}, f_{i}, t_{i}, t_{i}^{-1}$ (for $i=0, \ldots, e-1$ ) and $\partial$ and relations given in $[2, \S 6.1]$. We denote by $\mathcal{U}_{q}^{\prime}\left(\widehat{\mathfrak{s l}_{e}}\right)$ the subalgebra generated by $e_{i}, f_{i}, t_{i}, t_{i}^{-1}$ (for $i=0, \ldots, e-1$ ). For $i=0, \ldots, e-1$, we denote by $\Lambda_{i}$ the fundamental weights and the simple roots are given by:

$$
\alpha_{i}=-\Lambda_{i-1}+2 \Lambda_{i}-\Lambda_{i+1}
$$

where the indices are taken modulo $e$. There is an action of $\mathcal{U}_{q}^{\prime}\left(\widehat{\mathfrak{s l}}_{e}\right)$ on the Fock space. This action depends on the choice of $\mathbf{s}$ and the module generated by the empty multipartition is an irreducible highest weight module with highest weight $\Lambda_{s_{1}}+\cdots+\Lambda_{s_{l}}$. We do not need the precise definition of this action and we refer to [2, Ch. 6] for details. 
To each $\boldsymbol{\lambda} \in \Pi^{l}(n)$ is associated its Young tableau:

$$
[\boldsymbol{\lambda}]=\left\{(a, b, c) \mid a \geqslant 1, c \in\{0, \ldots, l-1\}, 1 \leqslant b \leqslant \lambda_{a}^{c}\right\} .
$$

We define the content of a node $\gamma=(a, b, c) \in[\boldsymbol{\lambda}]$ as follows:

$$
\operatorname{cont}(\gamma)=b-a+s_{c}
$$

and the residue res $(\gamma)$ is by definition the content of the node taken modulo $e$. We will say that $\gamma$ is an $i+e \mathbb{Z}$-node of $\boldsymbol{\lambda}$ when $\operatorname{res}(\gamma) \equiv i+e \mathbb{Z}$ (we will sometimes simply called it an $i$-node). Finally, We say that $\gamma$ is removable when $\gamma=(a, b, c) \in[\boldsymbol{\lambda}]$ and $[\boldsymbol{\lambda}] \backslash\{\gamma\}$ is the Young diagram of an $l$-partition. Similarly, $\gamma$ is addable when $\gamma=(a, b, c) \notin[\boldsymbol{\lambda}]$ and $[\boldsymbol{\lambda}] \cup\{\gamma\}$ is the Young diagram of an $l$-partition.

Let $\gamma, \gamma^{\prime}$ be two removable or addable $i$-nodes of $\boldsymbol{\lambda}$. We denote

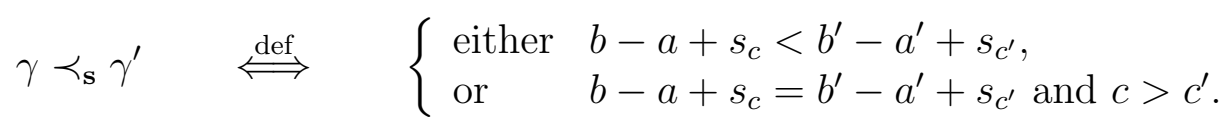

For $\boldsymbol{\lambda}$ an $l$-partition and $i \in \mathbb{Z} / e \mathbb{Z}$, we can consider its set of addable and removable $i$-nodes. Let $w_{i}^{(e, \mathbf{s})}(\boldsymbol{\lambda})$ be the word obtained first by writing the addable and removable $i$-nodes of $\boldsymbol{\lambda}$ in increasing order with respect to $\prec_{\mathbf{s}}$, next by encoding each addable $i$-node by the letter $A$ and each removable $i$-node by the letter $R$. Write $\widetilde{w}_{i}^{(e, s}(\boldsymbol{\lambda})=A^{p} R^{q}$ for the word derived from $w_{i}^{(e, \mathbf{s})}(\boldsymbol{\lambda})$ by deleting as many of the factors $R A$ as possible. In the following, we will sometimes write $\widetilde{w}_{i}(\boldsymbol{\lambda})$ and $w_{i}(\boldsymbol{\lambda})$ instead of $\widetilde{w}_{i}^{(e, \mathbf{s})}(\boldsymbol{\lambda})$ and $w_{i}^{(e, \mathbf{s})}(\boldsymbol{\lambda})$ if there is no possible confusion.

If $p>0$, let $\gamma$ be the rightmost addable $i$-node in $\widetilde{w}_{i}$. The node $\gamma$ is called the good addable $i$-node. If $r>0$, the leftmost removable $i$-node in $\widetilde{w}_{i}$ is called the good removable $i$-node.

Example 1. For $l=2, \mathbf{s}=(0,1)$ and $e=3$. Let us consider the 2-partition $\boldsymbol{\lambda}:=$ $((4),(2,1))$ of 7 . We write its Young tableau and the residues of the nodes in the associated boxes:

$$
\left(\begin{array}{l|l|l|l|l|l|}
\hline 0 & 1 & 2 & 0 \\
\hline
\end{array}, \begin{array}{ll}
\hline & 2 \\
\hline 0 &
\end{array}\right)
$$

We have $\widetilde{w}_{0}(\boldsymbol{\lambda})=R A R$ and thus $(1,4,1)$ is a good removable 0 -node for $\boldsymbol{\lambda}$. We have $\widetilde{w}_{2}(\boldsymbol{\lambda})=A A R$ and thus $(2,1,1)$ is a good addable 2-node for $\boldsymbol{\lambda}$ and $(1,2,2)$ is a good removable 2 -node for it.

We denote by $\mathcal{G}_{e, \mathbf{s}}$ the crystal of the Fock space computed using the Kashiwara operators $\widetilde{e}_{i}^{e, \mathbf{s}}$ and $\widetilde{f}_{i}^{e, \mathbf{s}}$. Again, we refer to [2] for details. This is the graph with

- vertices : the $l$-partitions $\boldsymbol{\lambda} \vdash_{l} n$ with $n \in \mathbb{Z}_{\geqslant 0}$.

- arrows: $\boldsymbol{\lambda} \stackrel{i}{\rightarrow} \boldsymbol{\mu}$ if and only if $\widetilde{f}_{i}^{e, \mathbf{s}} \boldsymbol{\lambda}=\boldsymbol{\mu}$ (or equivalently $\widetilde{e}_{i}^{e, \mathbf{s}} \boldsymbol{\mu}=\boldsymbol{\lambda}$ ). This means that $\boldsymbol{\mu}$ is obtained by adding to $\boldsymbol{\lambda}$ the good addable $i$-node, or equivalently, $\boldsymbol{\lambda}$ is obtained from $\boldsymbol{\mu}$ by removing the good removable $i$-node. 
Example 2. For $l=3, e=2$ and $\mathbf{s}=(0,0,1)$ the graph below is the subgraph of $\mathcal{G}_{e, \mathbf{s}}$ containing the empty 3 -partition and with the 3 -partitions with rank less or equal than 4.

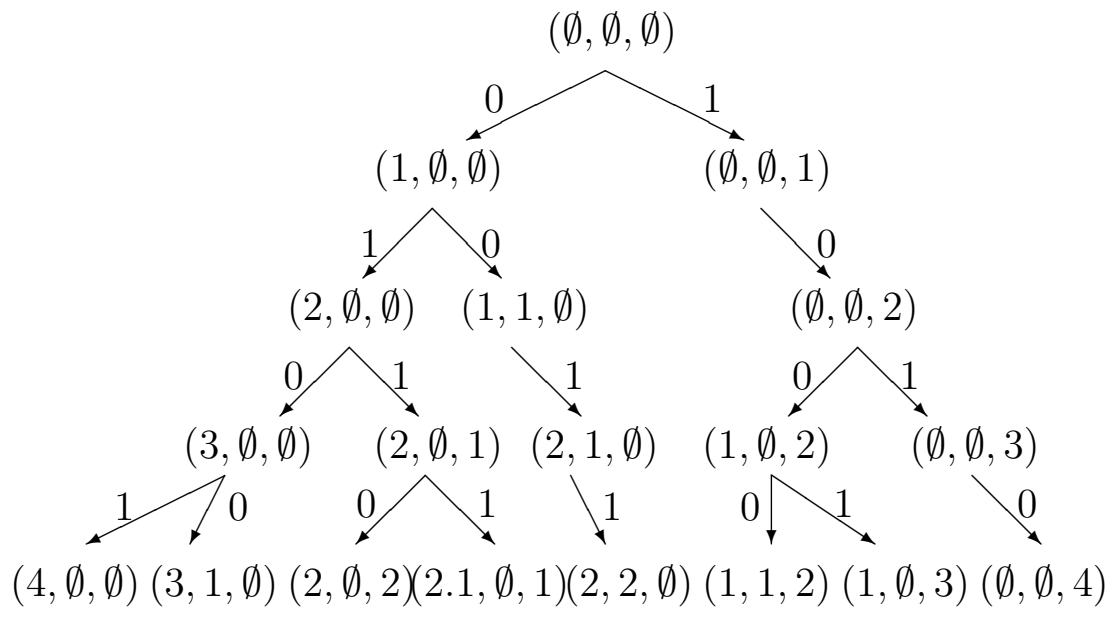

Let $\Phi_{e, \mathbf{s}}(n)$ be the set of $l$-partitions of rank $n$ in the connected component of $\mathcal{G}_{e, \mathbf{s}}$ containing the empty l-partition. This is called the set of Uglov l-partitions. Hence, by definition, an Uglov $l$-partition is defined by adding successively good nodes to the empty $l$-partition (with arbitrary residues). It strongly depends on the choice of s. Assume that $\mathbf{s}$ is such that $0<s_{j}-s_{i}<e$ for all $0<i<j \leqslant l$ then the set $\Phi_{e, \mathbf{s}}(n)$ is known as the set of FLOTW $l$-partitions and it has a nice non recursive description (see $[2, \S 6.3 .2]$ ). We have $\boldsymbol{\lambda}=\left(\lambda^{1}, \ldots, \lambda^{l}\right) \in \Phi_{\mathbf{s}, e}(n)$ if and only if:

1. For all $j=1, \ldots, l-1$ and $i \in \mathbb{Z}_{>0}$, we have:

$$
\lambda_{i}^{j} \geqslant \lambda_{i+s_{j+1}-s_{j}}^{j+1}
$$

2. For all $i \in \mathbb{Z}_{>0}$, we have:

$$
\lambda_{i}^{l} \geqslant \lambda_{i+e+s_{1}-s_{l}}^{1}
$$

3. For all $k \in \mathbb{Z}_{>0}$, the set

$$
\left\{\lambda_{i}^{j}-i+s_{j}+e \mathbb{Z} \mid i \in \mathbb{Z}_{>0}, \lambda_{i}^{j}=k, j=1, \ldots, l\right\},
$$

is a proper subset of $\mathbb{Z} / e \mathbb{Z}$.

In general, we do not have such a nice description of the set of Uglov $l$-partitions.

Example 3. In the case where $l=1$, the set $\Phi_{e,(0)}(n)$ is the set of $e$-regular partitions of $n$, that is, the set of partitions of rank $n$ such that no non zero parts are repeated $e$ or more times.

Example 4. Following Example 2, we have

$$
\Phi_{2,(0,0,1)}(4)=\{(4, \emptyset, \emptyset),(3,1, \emptyset),(2, \emptyset, 2),(2.1, \emptyset, 1),(2,2, \emptyset),(1,1,2),(1, \emptyset, 3),(\emptyset, \emptyset, 4)\}
$$




\subsection{Crystal isomorphisms}

In this part, we recall the definition of certain crystal isomorphisms studied in [9]. These maps will be intensively used in the next sections.

Let $\widehat{\mathfrak{S}}_{l}$ be the (extended) affine symmetric group. This is defined as follows. We denote by $P_{l}:=\mathbb{Z}^{l}$ the $\mathbb{Z}$-module with standard basis $\left\{y_{i} \mid i=1, \ldots, l\right\}$. For $i=1, \ldots, l-1$, we denote by $\sigma_{i}$ the transposition $(i, i+1)$ of $\mathfrak{S}_{l}$. Then $\widehat{\mathfrak{S}}_{l}$ can be seen as the semidirect product $P_{l} \rtimes \mathfrak{S}_{l}$ where the relations are given by $\sigma_{i} y_{j}=y_{j} \sigma_{i}$ for $j \neq i, i+1$ and $\sigma_{i} y_{i} \sigma_{i}=y_{i+1}$ for $i=1, \ldots, l-1$ and $j=1, \ldots, l$. This group acts on $\mathbb{Z}^{l}$ by setting for any $\mathbf{s}=\left(s_{1}, \ldots, s_{l}\right) \in \mathbb{Z}^{l}$ :

$$
\begin{aligned}
\sigma_{c} . \mathbf{s} & =\left(s_{1}, \ldots, s_{c-1}, s_{c+1}, s_{c}, s_{c+2}, \ldots, s_{l}\right) & & \text { for } c=1, \ldots, l-1 \text { and } \\
y_{i} . \mathbf{s} & =\left(s_{1}, s_{2}, \ldots, s_{i}+e, \ldots, s_{l}\right) & & \text { for } i=1, \ldots, l
\end{aligned}
$$

A fundamental domain for this action is given by

$$
\mathcal{A}_{l}^{e}:=\left\{\left(s_{1}, \ldots, s_{l}\right) \in \mathbb{Z}^{l} \mid 0 \leqslant s_{1} \leqslant \cdots \leqslant s_{l}<e\right\} .
$$

Note that we thus have a description of $\Phi_{\mathbf{s}, e}(n)$ when $\mathbf{s}$ is in this domain by $\S 2.1$. Let $\tau:=y_{l} \sigma_{l-1} \ldots \sigma_{1}$ then we see that $\widehat{\mathfrak{S}}_{l}$ is generated by $\tau$ and $\sigma_{i}$ for $i=1, \ldots, l-1$. In addition, we have:

$$
\tau . \mathbf{s}=\left(s_{2}, \ldots, s_{l}, s_{1}+e\right) .
$$

Assume that $\mathbf{s} \in \mathbb{Z}^{l}$ and $\mathbf{s}^{\prime} \in \mathbb{Z}^{l}$ are in the same orbit modulo $\widehat{\mathfrak{S}}_{l}$. As explained in $[2$, $\S 6.2 .17]$, the crystal graph theory allows to construct a combinatorial bijection between the two sets of Uglov $l$-partitions $\Phi_{\mathbf{s}, e}(n)$ and $\Phi_{\mathbf{s}^{\prime}, e}(n)$. Let $\boldsymbol{\lambda} \in \Phi_{\mathbf{s}, e}(n)$ then there exists a sequence $\left(i_{1}, \ldots, i_{n}\right) \in(\mathbb{Z} / e \mathbb{Z})^{n}$ such that:

$$
\tilde{f}_{i_{1}}^{e, \mathbf{s}} \ldots \tilde{f}_{i_{n}}^{e, \mathbf{s}} \emptyset=\boldsymbol{\lambda}
$$

Then there exists $\boldsymbol{\mu} \in \Phi_{\mathbf{s}^{\prime}, e}(n)$ such that

$$
\widetilde{f}_{i_{1}}^{e, \mathbf{s}^{\prime}} \ldots \widetilde{f}_{i_{n}}^{e, \mathbf{s}^{\prime}} \emptyset=\boldsymbol{\mu}
$$

We set $\Psi_{\mathbf{s} \rightarrow \mathbf{s}^{\prime}}^{e}(\boldsymbol{\lambda}):=\boldsymbol{\mu}$ (it does not depends on the choice of the sequence $\left(i_{1}, \ldots, i_{n}\right)$ ). This defines a bijection

$$
\Psi_{\mathbf{s} \rightarrow \mathbf{s}^{\prime}}^{e}: \Phi_{\mathbf{s}, e}(n) \rightarrow \Phi_{\mathbf{s}^{\prime}, e}(n) .
$$

A combinatorial description of this map is given in [9]. Let us quickly explain how it works. There exists $w \in \widehat{\mathfrak{S}}_{l}$ such that $\mathbf{s}^{\prime}=w \cdot \mathbf{s}$. Then, $w$ is a product of $\tau$ and $\sigma_{i}$ 's $(i=1, \ldots, l-1)$. Thus $\Psi_{\mathbf{s} \rightarrow \mathbf{s}^{\prime}}^{e}$ is a composition of maps of the form $\Psi_{\mathbf{v} \rightarrow \tau . \mathbf{v}}^{e}$ and $\Psi_{\mathbf{v} \rightarrow \sigma_{i} . \mathbf{v}}^{e}$ with $\mathbf{v} \in \mathbb{Z}^{l}$ make explicit by induction.

- For all $\boldsymbol{\lambda}=\left(\lambda^{1}, \ldots, \lambda^{l}\right) \in \Phi_{e, \mathbf{v}}(n)$, we have

$$
\Psi_{\mathbf{v} \rightarrow \tau . \mathbf{v}}^{e}(\boldsymbol{\lambda})=\left(\lambda^{2}, \ldots, \lambda^{l}, \lambda^{1}\right)
$$


- For all $\boldsymbol{\lambda}=\left(\lambda^{1}, \ldots, \lambda^{l}\right) \in \Phi_{e, \mathbf{v}}(n)$, we have

$$
\Psi_{\mathbf{v} \rightarrow \sigma_{i} . \mathbf{v}}^{e}(\boldsymbol{\lambda})=\left(\lambda^{1}, \ldots, \lambda^{i-1}, \tilde{\lambda}^{i+1}, \tilde{\lambda}^{i}, \lambda^{i+2}, \ldots, \lambda^{l}\right)
$$

where $\left(\widetilde{\lambda}^{i+1}, \widetilde{\lambda}^{i}\right)$ is obtained from $\left(\lambda^{i}, \lambda^{i+1}\right)$ via a simple purely combinatorial process described in [9, th. 5.4.2] (in terms of Lusztig symbols) or in [8, §5.3] (in terms of Young tableaux).

Assume that $\mathbf{s}=\left(s_{1}, \ldots, s_{l}\right)$ satisfies $s_{i}-s_{j} \geqslant n-1-e$ for all $i<j$ then we say that $\mathbf{s}$ is very dominant. If both $\mathbf{s}$ and $\mathbf{s}^{\prime}$ are very dominant (comparing to $n$ ) and in the same orbit then $\Psi_{\mathbf{s} \rightarrow \mathbf{s}^{\prime}}^{e}$ restricted to $\Phi_{\mathbf{s}, e}(n)$ is the identity and the set $\Phi_{\mathbf{s}, e}(n)$ is known as the set of Kleshchev $l$-partitions (see [2, Ex. 6.2.16]).

If $\mathbf{s} \in \mathbb{Z}^{l}$, one way to compute the set $\Phi_{\mathbf{s}, e}(n)$ of Uglov $l$-partitions consists in finding $w \in \widehat{\mathfrak{S}}_{l}$ such that $\mathbf{s}^{\prime}=w . \mathbf{s} \in \mathcal{A}_{l}^{e}$. We can then use the description of the set $\Phi_{\mathbf{s}^{\prime}, e}(n)$ in $\S 2.1$ and then apply the isomorphism $\Psi_{\mathbf{s}^{\prime} \rightarrow \mathbf{s}}^{e}$.

Example 5. For $l=3, e=2$ and $\mathbf{s}=(2,0,3)$ the graph below is the subgraph of $\mathcal{G}_{e, \mathbf{s}}$ containing the empty 3-partition.

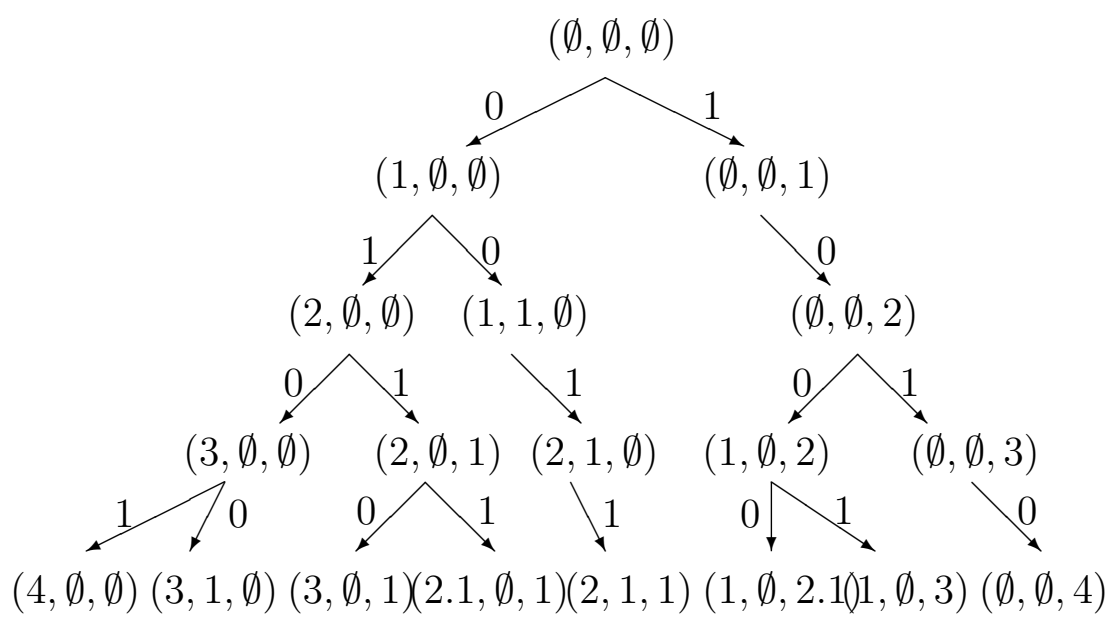

Looking at example 2, we see that $\Psi_{(0,0,1) \rightarrow(2,0,3)}^{2}$ is the identity for the Uglov 3partitions of ranks $\leqslant 3$, and we have

$$
\begin{gathered}
\Psi_{(0,0,1) \rightarrow(2,0,3)}^{2}(2, \emptyset, 2)=(3, \emptyset, 1), \Psi_{(0,0,1) \rightarrow(2,0,3)}^{2}(2,2, \emptyset)=(2,1,1), \\
\Psi_{(0,0,1) \rightarrow(2,0,3)}^{2}(1,1,2)=(1, \emptyset, 2.1)
\end{gathered}
$$

and $\Psi_{(0,0,1) \rightarrow(2,0,3)}^{3}$ is the identity for the others Uglov 3-partitions of ranks 4 . This formula can be also obtained without looking at the crystal. Indeed, we have $(2,0,3)=$ $\left(\sigma_{1} \tau\right)^{2}(0,0,1)$ and thus

$$
\Psi_{(0,0,1) \rightarrow(2,0,3)}^{2}=\Psi_{(0,0,1) \rightarrow \tau(0,0,1)}^{2} \circ \Psi_{\tau(0,0,1) \rightarrow \sigma_{1} \tau(0,0,1)}^{2} \circ \Psi_{\sigma_{1} \tau(0,0,1) \rightarrow \tau \sigma_{1} \tau(0,0,1)}^{3} \circ \Psi_{\tau \sigma_{1} \tau(0,0,1) \circ \sigma_{1} \tau \sigma_{1} \tau(0,0,1)}^{2}
$$

and one can use the combinatorial description of the isomorphisms. 


\section{Two maps on crystals}

The above results will allow us to recover and give precisions on two results on crystals in affine type $A$ thanks to quite elementary proofs. These two results concern a particular choice of multicharge which naturally appears in the context of Hecke algebras, as we will see in the next part. In this section, we thus assume that $l$ divides $e$ and that $\mathbf{s} \in \mathbb{Z}^{l}$ is in the orbit of the multicharge $(0, e / l, \ldots,(l-1) e / l)$ modulo $\widehat{\mathfrak{S}}_{l}$.

\subsection{Hu's map}

The first result that we want ro recover is in fact a direct generalization of a result by $\mathrm{Hu}$ [4, Theorem 3.6]. We propose here an elementary proof of this result using our crystal isomorphisms and we will also give a general method to explicitly compute the $l$-partitions that this results allows to define. Part of this result (Lemma 7 below) is in fact contained in the $\mathrm{PhD}$ thesis of the author [7] and used in [5].

Proposition $6(\mathrm{Hu})$. Assume that $\boldsymbol{\lambda} \in \Phi_{e, \mathbf{s}}(n)$. Then there exists a sequence $\left(i_{1}, \ldots, i_{n}\right) \in$ $(\mathbb{Z} / e \mathbb{Z})^{n}$ such that:

$$
\tilde{f}_{i_{1}}^{e, \mathbf{s}} \ldots \tilde{f}_{i_{n}}^{e, \mathbf{s}} \emptyset=\boldsymbol{\lambda}
$$

Then for any such sequence, there exists $\boldsymbol{\mu} \in \Phi_{e, \mathbf{s}}(n)$ such that

$$
\widetilde{f}_{i_{1}+e / l}^{e, \mathbf{s}} \ldots \widetilde{f}_{i_{n}+e / l}^{e, \mathbf{s}} \emptyset=\boldsymbol{\mu}
$$

To prove this proposition, we will proceed in two steps, we first prove the proposition for a particular choice of multicharge which is in $\mathcal{A}_{l}^{e}$, and then make use of the crystal isomorphisms we have already defined.

Lemma 7. Let $\mathbf{s}=(0, e / l, \ldots,(l-1) e / l)$ Assume that $\boldsymbol{\lambda}=\left(\lambda^{1}, \ldots, \lambda^{l}\right) \in \Phi_{e, \mathbf{s}}(n)$ and that we have a sequence $\left(i_{1}, \ldots, i_{n}\right) \in(\mathbb{Z} / e \mathbb{Z})^{n}$ such that:

$$
\tilde{f}_{i_{1}}^{e, \mathbf{s}} \ldots \tilde{f}_{i_{n}}^{e, \mathbf{s}} \emptyset=\lambda
$$

Then we have that $\boldsymbol{\mu}:=\left(\lambda^{l}, \lambda^{1}, \ldots, \lambda^{l-1}\right) \in \Phi_{e, \mathbf{s}}(n)$ and we have

$$
\widetilde{f}_{i_{1}+e / l}^{e, \mathbf{s}} \ldots \widetilde{f}_{i_{n}+e / l}^{e, \mathbf{s}} \emptyset=\boldsymbol{\mu} .
$$

Proof. We argue by induction on $n$. The lemma is clear for the empty $l$-partition. Assume now that $n>0$. Let $\boldsymbol{\lambda} \in \Phi_{e, \mathbf{s}}(n)$ and assume that we have a sequence $\left(i_{1}, \ldots, i_{n}\right) \in$ $(\mathbb{Z} / e \mathbb{Z})^{n}$ such that

$$
\widetilde{f}_{i_{1}}^{e, \mathbf{s}} \ldots \tilde{f}_{i_{n}}^{e, \mathbf{s}} \emptyset=\lambda
$$

Set $\boldsymbol{\lambda}^{\prime}:=\widetilde{f}_{i_{2}}^{e, \mathbf{s}} \ldots \tilde{f}_{i_{n}}^{e, \mathbf{s}} \emptyset$ then $\boldsymbol{\lambda}^{\prime}=\left(\lambda^{\prime 1}, \ldots, \lambda^{\prime l}\right)$ is in $\Phi_{e, \mathbf{s}}(n-1)$ and by induction, $\boldsymbol{\mu}^{\prime}:=$ $\left(\lambda^{\prime l}, \lambda^{\prime 1}, \ldots, \lambda^{\prime l-1}\right)$ is in $\Phi_{e, \mathbf{s}}(n-1)$ and we have $\boldsymbol{\mu}^{\prime}=\widetilde{f}_{i_{2}+e / l}^{e, \mathbf{s}} \ldots \widetilde{f}_{i_{n}+e / l}^{e, \mathbf{s}} \emptyset$.

Now, by hypothesis, we have that $\gamma=[\boldsymbol{\lambda}] /\left[\boldsymbol{\lambda}^{\prime}\right]$ is a good addable $i_{1}$-node for $\boldsymbol{\lambda}^{\prime}$ (and $(e, \mathbf{s}))$. Set $(a, b, c):=\gamma$. Then $\gamma^{\prime}:=[\boldsymbol{\mu}] /\left[\boldsymbol{\mu}^{\prime}\right]$ is an addable node for $\boldsymbol{\mu}^{\prime}$. We have 
$\gamma^{\prime}=(a, b, c+1)$ (where the 3rd component is understood modulo $l$ ) and, by our choice of multicharge, it is a $i_{1}+e / l$-node. We want to show that this is a good addable node for $\boldsymbol{\mu}^{\prime}$.

Assume that $\gamma_{1}=\left(a_{1}, b_{1}, c_{1}\right)$ is a $i_{1}$-addable or removable node for $\boldsymbol{\lambda}^{\prime}$. Then $\gamma_{1}^{\prime}:=$ $\left(a_{1}, b_{1}, c_{1}+1\right)$ is an addable or removable node for $\boldsymbol{\mu}^{\prime}$ (and it is removable, resp. addable, if and only if $\gamma_{1}$ is). We have that

$$
\operatorname{cont}\left(\gamma_{1}^{\prime}\right)=\left\{\begin{aligned}
\operatorname{cont}\left(\gamma_{1}\right)+e / l & \text { if } c_{1} \neq l \\
\operatorname{cont}\left(\gamma_{1}\right)+e / l-e & \text { if } c_{1}=l
\end{aligned}\right.
$$

Thus, we have $\gamma_{1} \prec_{e, \mathbf{s}} \gamma$ if and only if $\gamma_{1}^{\prime} \prec_{e, \mathbf{s}} \gamma^{\prime}$.

Reciprocally, if $\gamma_{1}^{\prime}=\left(a_{1}, b_{1}, c_{1}\right)$ is a $i_{n}+e / l$-addable or removable node for $\boldsymbol{\mu}^{\prime}$. Then $\gamma_{1}:=\left(a_{1}, b_{1}, c_{1}-1\right)$ is an addable or removable for $i_{1}$-node for $\boldsymbol{\lambda}^{\prime}$ (and it is removable, resp. addable, if and only if $\gamma_{1}^{\prime}$ is $)$. We have that $\gamma_{1} \prec_{e, \mathbf{s}} \gamma$ if and only if $\gamma_{1}^{\prime} \prec_{e, \mathbf{s}} \gamma^{\prime}$. Thus we have $w_{i_{1}}^{(e, \mathbf{s})}\left(\boldsymbol{\lambda}^{\prime}\right)=w_{i_{1}+e / l}^{(e, \mathbf{s})}\left(\boldsymbol{\mu}^{\prime}\right)$.

This discussion implies that $\gamma^{\prime}$ is good addable $i_{1}+e / l$-node for $\boldsymbol{\mu}^{\prime}$ and thus that

$$
\tilde{f}_{i_{1}+e / l}^{e, \mathbf{s}} \ldots \widetilde{f}_{i_{n}+e / l}^{e, \mathbf{s}} \emptyset=\boldsymbol{\mu},
$$

as required.

We can now give a proof of Proposition 6. Assume that $\mathbf{s} \in \mathbb{Z}^{l}$ is in the orbit of $(0, e / l, \ldots,(l-1) e / l)$ modulo $\widehat{\mathfrak{S}}_{l}$. Let $\boldsymbol{\lambda} \in \Phi_{e, \mathbf{s}}(n)$ and consider a sequence $\left(i_{1}, \ldots, i_{n}\right) \in$ $(\mathbb{Z} / e \mathbb{Z})^{n}$ such that:

$$
\widetilde{f}_{i_{1}}^{e, \mathbf{s}} \ldots \widetilde{f}_{i_{n}}^{e, \mathbf{s}} \emptyset=\boldsymbol{\lambda} .
$$

Set $\mathbf{s}^{\prime}:=(0, e / l, \ldots,(l-1) e / l)$, then by definition, we have

$$
\widetilde{f}_{i_{1}}^{e, \mathbf{s}^{\prime}} \ldots \widetilde{f}_{i_{n}}^{e, \mathbf{s}^{\prime}} \emptyset=\Psi_{\mathbf{s} \rightarrow \mathbf{s}^{\prime}}^{e}(\boldsymbol{\lambda}) .
$$

We can thus use Lemma 7 to deduce that there exists $\boldsymbol{\mu}^{\prime} \in \Phi_{e, \mathbf{s}^{\prime}}(n)$ such that

$$
\widetilde{f}_{i_{1}+e / l}^{e, \mathbf{s}^{\prime}} \cdots \widetilde{f}_{i_{n}+e / l}^{e, \mathbf{s}^{\prime}} \emptyset=\boldsymbol{\mu}^{\prime},
$$

and using again our crystal isomorphism, we get that:

$$
\widetilde{f}_{i_{1}+e / l}^{e, \mathbf{s}} \ldots \widetilde{f}_{i_{n}+e / l}^{e, \mathbf{s}} \emptyset=\Psi_{\mathbf{s}^{\prime} \rightarrow \mathbf{s}}^{e}\left(\boldsymbol{\mu}^{\prime}\right),
$$

so the result follows. Note in addition that the $l$-partition $\boldsymbol{\mu}^{\prime}$ may be explicitly described thanks to the description of the crystal isomorphism without the computation of the crystal itself.

Example 8. Take $l=2$ and assume that $e=4$. We set $\mathbf{s}=(0,10)$ which is in the orbit of $\mathbf{s}^{\prime}=(0,2) \in \mathcal{A}_{2}^{4}$. We take $\boldsymbol{\lambda}=(1.1,5.1) \in \Phi_{(0,10)}^{4}(8)$. Note that the multicharge is very dominant for 2-partitions of rank 8 so $(1.1,5.1)$ is a Kleshchev bipartition. If we want to find the bipartition $\boldsymbol{\mu}$ of Proposition 6, we first need to find the bipartition $\boldsymbol{\lambda}^{\prime} \in \Phi_{(0,2)}^{4}(8)$ such that $\Psi_{\mathbf{s} \rightarrow \mathbf{s}^{\prime}}^{4}(\boldsymbol{\lambda})=\boldsymbol{\lambda}^{\prime}$. Using our description of the isomorphisms, we get $\boldsymbol{\lambda}^{\prime}=(2.1,5)$. We then have $\boldsymbol{\mu}=\Psi_{\mathrm{s}^{\prime} \rightarrow \mathbf{s}}^{4}(5,2.1)=(4,3.1)$. 


\subsection{The map $\iota_{k}^{\mathrm{s}}$}

We keep the hypothesis that $\mathbf{s}$ is in the orbit of the multicharge $(0, e / l, \ldots,(l-1) e / l)$ modulo $\widehat{\mathfrak{S}}_{l}$. We now consider another map defined using the crystal. As explained in [6], its existence follows from [10] in the case where $l=2$. Here we will give a general version which can be seen in [5] and we will again give a different easy proof using our crystal isomorphism. We will also make things more explicit.

Proposition 9. Let $k$ be an integer dividing $l$. Set $\mathbf{v}:=(0, e / l, \ldots, e(k-1) / l) \in \mathbb{Z}^{k}$. There exists a unique map

$$
\iota_{k}^{\mathbf{s}}: \Phi_{k e / l, \mathbf{v}}(n) \rightarrow \Phi_{e, \mathbf{s}}(\ln / k)
$$

well-defined as follows. For all $\boldsymbol{\lambda} \in \Phi_{k e / l, \mathbf{v}}(n)$, there exists $\left(i_{1}, \ldots, i_{n}\right) \in \mathbb{Z}^{n}$ such that

$$
\tilde{f}_{i_{1}}^{k e / l, \mathbf{v}} \ldots \tilde{f}_{i_{n}}^{k e / l, \mathbf{v}} \emptyset=\boldsymbol{\lambda},
$$

(the indices are understood modulo ke/l.) Then for all such sequences, we have:

$$
\underbrace{\widetilde{f}_{i_{1}}^{e, \mathbf{s}} \widetilde{f}_{i_{1}+k e / l}^{e, \mathbf{s}} \cdots \widetilde{f}_{i_{1}+e-k e / l}^{e, \mathbf{s}}}_{l / k} \cdots \underbrace{\widetilde{f}_{i_{n}, \mathbf{s}} \widetilde{f}_{i_{n}+k e / l}^{e, \mathbf{s}} \cdots \widetilde{f}_{i_{n}+e-k e / l}^{e, \mathbf{s}}}_{l / k} \emptyset=\iota_{k}^{\mathbf{s}}(\boldsymbol{\lambda})
$$

(the indices are understood modulo e.)

In the same spirit as the last result, our strategy consists in proving the result when $\mathbf{s}$ is in the fundamental domain $\mathcal{A}_{l}^{e}$.

Lemma 10. Let $k$ be an integer dividing $l$. Set $\mathbf{s}=(0, e / l, \ldots,(l-1) e / l)$ and $\mathbf{v}:=$ $(0, e / l, \ldots, e(k-1) / l) \in \mathbb{Z}^{k}$. There exists a unique map

$$
\iota_{k}^{\mathbf{s}}: \Phi_{k e / l, \mathbf{v}}(n) \rightarrow \Phi_{e, \mathbf{s}}(\ln / k)
$$

well-defined as follows. For all $\boldsymbol{\lambda} \in \Phi_{k e / l, \mathbf{v}}(n)$, there exists $\left(i_{1}, \ldots, i_{n}\right) \in \mathbb{Z}^{n}$ such that

$$
\tilde{f}_{i_{1}}^{k e / l, \mathbf{v}} \ldots \tilde{f}_{i_{n}}^{k e / l, \mathbf{v}} \emptyset=\lambda
$$

Then for all such sequences, we have:

$\underbrace{\tilde{f}_{i_{1}}^{e, \mathbf{s}} \tilde{f}_{i_{1}+k e / l}^{e, \mathbf{s}} \cdots \tilde{f}_{i_{1}+e-k e / l}^{e, \mathbf{s}}}_{l / k} \cdots \underbrace{\tilde{f}_{i_{n}}^{e, \mathbf{s}} \tilde{f}_{i_{n}+k e / l}^{e, \mathbf{s}} \ldots \tilde{f}_{i_{n}+e-k e / l}^{e, \mathbf{s}}}_{l / k} \emptyset=(\underbrace{\lambda^{1}, \ldots, \lambda^{k}}_{l / k}, \underbrace{\lambda^{1}, \ldots, \lambda^{k}}_{l / k}, \ldots, \underbrace{\lambda^{1}, \ldots, \lambda^{k}}_{l / k})$.

Proof. We again argue by induction on $n \in \mathbb{Z}_{\geqslant 0}$. The lemma is clear for the empty $l$-partition. Assume now that $n>0$. Let $\boldsymbol{\lambda} \in \Phi_{k e / l, \mathbf{v}}(n)$ and assume that we have a sequence $\left(i_{1}, \ldots, i_{n}\right) \in \mathbb{Z}^{n}$ such that

$$
\tilde{f}_{i_{1}}^{k e / l, \mathbf{v}} \ldots \tilde{f}_{i_{n}}^{k e / l, \mathbf{v}} . \emptyset=\boldsymbol{\lambda}
$$


Set $\boldsymbol{\lambda}^{\prime}:=\tilde{f}_{i_{2}}^{k e / l, \mathbf{v}} \ldots \tilde{f}_{i_{n}}^{k e / l, \mathbf{v}} . \emptyset$. By induction, we have:

$$
\underbrace{\widetilde{f}_{i_{1}}^{e, \mathbf{s}} \widetilde{f}_{i_{1}+k e / l}^{e, \mathbf{s}} \ldots \widetilde{f}_{i_{1}+e-k e / l}^{e, \mathbf{s}}}_{l / k} \ldots \underbrace{\widetilde{f}_{i_{n}}^{e, \mathbf{s}} \widetilde{f}_{i_{n}+k e / l}^{e, \mathbf{s}} \ldots \widetilde{f}_{i_{n}+e-k e / l}^{e, \mathbf{s}}}_{l / k} . \emptyset=\left(\lambda^{\prime 1}, \ldots, \lambda^{\prime k}, \lambda^{\prime 1}, \ldots, \lambda^{\prime k}, \ldots, \lambda^{\prime 1}, \ldots, \lambda^{\prime k}\right) .
$$

Denote

$$
\boldsymbol{\lambda}[0]:=\left(\lambda^{\prime 1}, \ldots, \lambda^{\prime k}, \lambda^{\prime 1}, \ldots, \lambda^{\prime k}, \ldots, \lambda^{\prime 1}, \ldots, \lambda^{\prime k}\right) .
$$

Set $\gamma=[\boldsymbol{\lambda}] /\left[\boldsymbol{\lambda}^{\prime}\right]$ and let $(a, b, c):=\gamma$. This is a good addable $i_{1}+(k e / l) \mathbb{Z}$-node for $\boldsymbol{\lambda}^{\prime}$. We have by definition $b-a+(c-1) e / l \equiv i_{1}+(k e / l) \mathbb{Z}$. So there exists $j \in\{0,1, \ldots, l / k-1\}$ such that $b-a+(c-1) e / l=i_{1}-j(k e / l)+e \mathbb{Z}$. We thus have $b-a+(c-1+(j-1) k) e / l=$ $i_{1}-(k e / l)+e \mathbb{Z}$. Let us denote $\gamma_{j}:=(a, b, c+(j-1) k)$ (where the 3rd component is understood modulo $l$ ). We have that the residue of $\gamma_{j}$ is $i_{1}-(k e / l)+e \mathbb{Z}$ for $\boldsymbol{\lambda}[0]$ and the multicharge $\mathbf{s}$.

Now assume that $\eta=\left(a^{\prime}, b^{\prime}, c^{\prime}\right)$ is an addable or a removable $i_{1}-(k e / l)+e \mathbb{Z}$-node for $\boldsymbol{\lambda}$, different from $\gamma$. As above, there exists $j^{\prime} \in\{1, \ldots, l\}$ such that $\eta_{j^{\prime}}:=\left(a^{\prime}, b^{\prime}, j^{\prime}-1\right)$ is an addable or removable $i_{1}-k e / l+e \mathbb{Z}$-node for $\boldsymbol{\lambda}[0]$ (and removable if and only if $\eta$ is).

In addition, by our definition of $\prec$, we have $\eta \prec_{\mathbf{v}} \gamma$ if and only is $\eta_{j^{\prime}} \prec_{\mathbf{s}} \gamma_{j}$. Reciprocally, all the $i_{1}-k e / l+e \mathbb{Z}$-nodes are obtained in this way.

This discussion implies that $\gamma_{j}$ is a good addable $i_{1}-k e / l$-node for $\boldsymbol{\lambda}[0]$ because $\gamma$ is a good one for $\boldsymbol{\lambda}$. We denote by $\boldsymbol{\lambda}[1]$ the $l$-partition obtained from this one by adding $\gamma_{j}$ to $\boldsymbol{\lambda}[0]$. We thus have $\boldsymbol{\lambda}[1]=\widetilde{f}_{i_{1}+e-k e / l}^{e, s} \boldsymbol{\lambda}^{\prime} \neq 0$.

Let us now consider $\gamma_{2}:=(a, b,(c-1+(j-2) k)$ ) (where the 3rd component is understood modulo $e$ ). It is an addable $i_{1}+(l-2) e / l+e \mathbb{Z}$-node for $\boldsymbol{\lambda}[1]$ and by exactly the same argument as above, we see that this is a good addable node. Let $\boldsymbol{\lambda}[2]$ be the $l$-partition obtained by adding this node to $\boldsymbol{\lambda}[1]$. We obtain $\widetilde{f}_{i_{1}+e-2 k e / l}^{e, \mathbf{s}} \widetilde{f}_{i_{1}+e-k e / l}^{e, \mathbf{s}} \boldsymbol{\lambda}^{\prime}=\boldsymbol{\lambda}[2]$. Continuing in this way we deduce $\widetilde{f}_{i_{1}}^{e, \mathbf{s}} \ldots \widetilde{f}_{i_{1}+(l-2) e / l}^{e, \mathbf{s}} \widetilde{f}_{i_{1}+(l-1) e / l}^{e, \mathbf{s}} \boldsymbol{\lambda}^{\prime}=\boldsymbol{\lambda}$, as required.

One can now give a general proof of the proposition. Assume that $\mathbf{s} \in \mathbb{Z}^{l}$ is in the orbit of $(0, e / l, \ldots,(l-1) e / l)$, that $\boldsymbol{\lambda} \in \Phi_{e, \mathbf{s}}(n)$ and that we have a sequence $\left(i_{1}, \ldots, i_{n}\right) \in \mathbb{Z}^{n}$ such that

$$
\tilde{f}_{i_{1}}^{k e / l, \mathbf{v}} \ldots \tilde{f}_{i_{n}}^{k e / l, \mathbf{v}} \emptyset=\boldsymbol{\lambda}
$$

Then, by the above lemma, if we set $\mathbf{s}^{\prime}=(0, e / l, \ldots,(l-1) e / l)$, we have

$\underbrace{\widetilde{f}_{i_{1}}^{e, \mathbf{s}^{\prime}} \tilde{f}_{i_{1}+k e / l}^{e, \mathbf{s}^{\prime}} \ldots \widetilde{f}_{i_{1}+e-k e / l}^{e, \mathbf{s}^{\prime}}}_{l / k} \cdots \underbrace{\widetilde{f}_{i_{n}}^{e, \mathbf{s}^{\prime}} \tilde{f}_{i_{n}+k e / l}^{e, \mathbf{s}^{\prime}} \ldots \widetilde{f}_{i_{n}+e-k e / l}^{e, \mathbf{s}^{\prime}}}_{l / k} \emptyset=\left(\lambda^{1}, \ldots, \lambda^{k}, \lambda^{1}, \ldots, \lambda^{k}, \ldots, \lambda^{1}, \ldots, \lambda^{k}\right)$

and thus one can conclude that

$$
\underbrace{\widetilde{f}_{i_{1}}^{e, \mathbf{s}} \widetilde{f}_{i_{1}+k e / l}^{e, \mathbf{s}} \ldots \widetilde{f}_{i_{1}+e-k e / l}^{e, \mathbf{s}}}_{l / k} \cdots \underbrace{\widetilde{f}_{i_{n}}^{e, s} \widetilde{f}_{i_{n}+k e / l}^{e, \mathbf{s}} \ldots \widetilde{f}_{i_{n}+e-k e / l}^{e, \mathbf{s}}}_{l / k} \emptyset=\Psi_{\mathbf{s}^{\prime} \rightarrow \mathbf{s}}^{e}\left(\lambda^{1}, \ldots, \lambda^{k}, \lambda^{1}, \ldots, \lambda^{k}, \ldots, \lambda^{1}, \ldots, \lambda^{k}\right) .
$$

which proves the theorem and also gives an explict way to compute the $l$-partition involved. 
Example 11. We take $l=2$ and $e=4$. We set $k=1$. Let $\lambda=(4.3 .1)$, this is a $e / 2$ regular partition and thus in $\Phi_{2,(0)}(8)$. By Lemma 10, we have $\iota_{1}^{(0,2)}(4.3 .1)=(4.3 .1,4.3 .1)$. Then we obtain for example $\iota_{1}^{(0,22)}(4.3 .1)=\Psi_{(0,2) \rightarrow(0,22)}^{4}(4.3 .1,4.3 .1)=(3.2 .1,4.3 .2 .1)$.

\section{Hecke algebras of type $G(p, p, n)$}

In this part, we apply the results above to recover and generalize some of the results of [4] and [6] and give precisions on them. We will freely use the results in [3].

\subsection{Definition}

Let $\eta \in \mathbb{C}^{\times}$. Assume that $n>2$. Let $\mathbf{s}=\left(s_{1}, \ldots, s_{l}\right) \in \mathbb{Z}^{l}$ and let $\eta \in \mathbb{C}^{\times}$. The cyclotomic Hecke algebra $\mathcal{H}_{n}(\mathbf{s})$ of type $G(l, 1, n)$ (also known as Ariki-Koike algebra) is the $\mathbb{C}$-algebra with a presentation by:

- generators : $\widetilde{T}_{0}, T_{1}, \ldots, T_{n-1}$,

- relations :

$$
\begin{aligned}
\left(\widetilde{T}_{0}-\eta^{s_{1}}\right) \ldots\left(\widetilde{T}_{0}-\eta^{s_{l}}\right) & =0 \\
\left(T_{i}-\eta\right)\left(T_{i}+1\right) & =0(1 \leqslant i \leqslant n-1) \\
\left(T_{0} T_{1}\right)^{2} & =\left(T_{1} T_{0}\right)^{2} \\
T_{i} T_{i+1} T_{i} & =T_{i+1} T_{i} T_{i+1}(1 \leqslant i<n) \\
T_{i} T_{j} & =T_{j} T_{i}(j \geqslant i+2) .
\end{aligned}
$$

The cyclotomic Hecke algebra $\mathcal{H}_{n}^{\prime}$ of type $G(l, l, n)$ is the $\mathbb{C}$-algebra with a presentation by :

- generators : $T_{0}, T_{1}, \ldots, T_{n-1}$,

- relations :

$$
\begin{array}{ll}
\left(T_{i}-\eta\right)\left(T_{i}+1\right)=0 & \text { for } 0 \leqslant i \leqslant n-1, \\
T_{i} T_{i+1} T_{i}=T_{i+1} T_{i} T_{i+1} & \text { for } 1 \leqslant i \leqslant n-2, \\
T_{0} T_{2} T_{0}=T_{2} T_{0} T_{2}, & \\
\left(T_{1} T_{0} T_{2}\right)^{2}=\left(T_{2} T_{1} T_{0}\right)^{2}, & \\
T_{0} T_{j}=T_{j} T_{0} & \text { for } j>2, \\
T_{i} T_{j}=T_{j} T_{i} & \text { for } i>0 \text { et } j>i+1, \\
\underbrace{T_{0} T_{1} T_{0} T_{1} \ldots}_{l \text { terms }}=\underbrace{T_{1} T_{0} T_{1} T_{0} \ldots}_{l \text { terms }} . &
\end{array}
$$

From now, we assume that $\eta$ is a primitive root of order $e>1$. Let $\mathbf{s} \in \mathbb{Z}^{l}$ be in the orbit of $(0, e / l, \ldots,(l-1) e / l)$ then the subalgebra of $\mathcal{H}_{n}:=\mathcal{H}_{n}(\mathbf{s})$ generated by

$$
\left\{T_{0}:=\widetilde{T}_{0}^{-1} T_{1} \widetilde{T}_{0}, T_{1}, \ldots, T_{n-1}\right\}
$$


is isomorphic to $\mathcal{H}_{n}^{\prime}$. Moreover $\mathcal{H}_{n}$ is $\mathbb{Z} / l \mathbb{Z}$-graded with respect to $\mathcal{H}_{n}^{\prime}$ with gradation

$$
\mathcal{H}_{n}=\bigoplus_{j=0}^{l-1} \widetilde{T}_{0}^{j} \mathcal{H}_{n}^{\prime} .
$$

As a consequence, one may use Clifford Theory to obtain results for the representation theory of $\mathcal{H}_{n}^{\prime}$ from the one of $\mathcal{H}_{n}$. To do this, we first need to recall some known results on the representation theory of $\mathcal{H}_{n}$

\subsection{Simple $\mathcal{H}_{n}$-modules}

The classification of the simple $\mathcal{H}_{n}$-modules that we need comes from the theory of basic sets. A complete review of this can be found in [2] but we quickly recall what we need here. One can define a certain set of finite-dimensional $\mathcal{H}_{n}$-modules which are parametrized by the set of $l$-partitions, they are called Specht modules

$$
\left\{S^{\boldsymbol{\lambda}} \mid \boldsymbol{\lambda} \in \Pi^{l}(n)\right\} .
$$

These modules are non simple (nor semisimple) in general but we have associated composition series. Let us denote by $\left[S^{\boldsymbol{\lambda}}: M\right]$ the multiplicity of $M \in \operatorname{Irr}\left(\mathcal{H}_{n}\right)$ in a composition series for $S^{\boldsymbol{\lambda}}$ (this is well-defined by the Jordan-Hölder theorem). Then the matrix defined by:

$$
\mathcal{D}:=\left(\left[S^{\boldsymbol{\lambda}}: M\right]\right)_{\boldsymbol{\lambda} \in \Pi^{l}(n), M \in \operatorname{Irr}\left(\mathcal{H}_{n}\right)}
$$

controls a part of the representation theory of $\mathcal{H}_{n}$. This is called the decomposition matrix.

We here follow $[2, \mathrm{Ch} .5, \mathrm{Ch} .6]$. Then one can define a pre-order $\ll_{\mathrm{s}}$ on the set of $l$ partitions which depends on the choice of $\mathbf{s}$. We don't give the definition of this pre-order here, all we need to know is the following theorem (see $[2, \S 6.7]$ ).

Theorem 12. Under the above hypotheses, for all $M \in \operatorname{Irr}\left(\mathcal{H}_{n}\right)$,

1. there exists $\boldsymbol{\lambda}_{M} \in \Phi_{\mathbf{s}, e}(n)$ such that $\left[S^{\boldsymbol{\lambda}_{M}}: M\right]=1$,

2. for all $\mu \vdash_{l} n$, if $\left[S^{\mu}: M\right] \neq 0$ then $\boldsymbol{\mu} \ll_{\mathbf{m}} \boldsymbol{\lambda}_{M}$.

The map $M \mapsto \boldsymbol{\lambda}_{M}$ is injective. As a consequence, if for all $M \in \operatorname{Irr}\left(\mathcal{H}_{n}\right)$ we denote $D_{\mathbf{s}, e}^{\lambda_{M}}:=M$, we have:

$$
\operatorname{Irr}\left(\mathcal{H}_{n}\right)=\left\{D_{\mathbf{s}, e}^{\boldsymbol{\mu}} \mid \boldsymbol{\mu} \in \Phi_{\mathbf{s}, e}(n)\right\}
$$

It is thus important to note that this theorem does not give one way to label the simple modules of the algebra $\mathcal{H}_{n}$ but in fact several ones: one for each choice of an element in the orbit of $\mathbf{s}$ modulo $\widehat{\mathfrak{S}}_{l}$. It is now natural to ask how all these parametrizations are connected. It turns out that the crystal isomorphisms make the links between them.

Proposition $13([8])$. Let $\mathbf{s} \in \mathbb{Z}^{l}$ and $\mathbf{s}^{\prime} \in \mathbb{Z}^{l}$ be two multicharges in the same orbit then for all $\boldsymbol{\lambda} \in \Phi_{\mathbf{s}, e}(n)$, we have $D_{\mathbf{s}, e}^{\boldsymbol{\lambda}}=D_{\mathbf{s}^{\prime}, e}^{\Psi_{\mathbf{s} \rightarrow \mathbf{s}^{\prime}}^{\mathbf{s}^{\prime}}(\boldsymbol{\lambda})}$. 


\subsection{Restriction of simple $\mathcal{H}_{n}$-modules}

We here set $\mathbf{s}=(0, e / l, \ldots,(l-1) e / l)$. There is a natural action of the cyclic group $\mathbb{Z} / l \mathbb{Z}$ on $\Pi^{l}(n)$ generated by the following map:

$$
\left(\lambda^{1}, \lambda^{2}, \ldots, \lambda^{l}\right) \mapsto\left(\lambda^{l}, \lambda^{1}, \ldots, \lambda^{l-1}\right) .
$$

For $\boldsymbol{\lambda} \in \Pi^{l}(n)$ we denote by $\widetilde{\boldsymbol{\lambda}}$ the associated equivalence class. Let

$$
r:=r(\boldsymbol{\lambda})=\frac{l}{\text { Cardinality of } \widetilde{\boldsymbol{\lambda}}} .
$$

The following theorem is proved in [3].

Theorem 14. Let $\boldsymbol{\lambda} \in \Phi_{\mathbf{s}, e}(n)$ then we have that $\operatorname{Res}\left(D_{\mathbf{s}, e}^{\boldsymbol{\lambda}}\right)$ is a direct sum of $r(\boldsymbol{\lambda})$ simple $\mathcal{H}_{n}^{\prime}$-modules.

It is also possible to show that if $\boldsymbol{\lambda}$ and $\boldsymbol{\mu}$ are in the same equivalence class then $\operatorname{Res}\left(D_{\mathbf{s}, e}^{\boldsymbol{\lambda}}\right)$ and $\operatorname{Res}\left(D_{\mathbf{s}, e}^{\boldsymbol{\mu}}\right)$ are isomorphic. In addition, the simple modules appearing in the restriction of the $D_{\mathbf{s}, e}^{\boldsymbol{\lambda}}$ 's determined the equivalence class of $\boldsymbol{\lambda}$. As a consequence, one can obtain a classification of the simple $\mathcal{H}_{n}$-modules knowing the numbers $r(\boldsymbol{\lambda})$. Applying Proposition 13 yields:

Proposition 15. Let $\mathbf{s}^{\prime} \in \mathbb{Z}^{l}$ in the same class as $\mathbf{s}=(0, e / l, \ldots,(l-1) e / l)$. Let $\boldsymbol{\lambda} \in$ $\Phi_{\mathbf{s}^{\prime}, e}(n)$ then we have that $\operatorname{Res}\left(D_{\mathbf{s}^{\prime}, e}^{\lambda}\right)$ is a direct sum of $r\left(\Psi_{\mathbf{s} \rightarrow, \mathbf{s}}^{e}(\boldsymbol{\lambda})\right)$ simple $\mathcal{H}_{n}^{\prime}$-modules.

The above proposition gives thus an explicit way to find the number of simple modules in the restriction of the simple $\mathcal{H}_{n}$-modules without refering to the notion of crystal and for all the known parametrization of the simples. This thus includes the usual parametrization by the set of Kleshchev $l$-partitions using our isomorphisms.

Proposition 16. Let $\mathbf{s}^{\prime} \in \mathbb{Z}^{l}$ in the same class as $\mathbf{s}=(0, e / l, \ldots,(l-1) e / l)$. Let $\boldsymbol{\lambda} \in$ $\Phi_{\mathbf{s}^{\prime}, e}(n)$ then $\operatorname{Res}\left(D_{\mathbf{s}^{\prime}, e}^{\boldsymbol{\lambda}}\right)$ splits into a sum of $x$ simple modules if and only if $\boldsymbol{\lambda} \in \operatorname{Im}\left(\iota_{l / x}^{\mathbf{s}^{\prime}}\right)$ and $\boldsymbol{\lambda} \notin \operatorname{Im}\left(\iota_{l / s}^{\mathbf{s}^{\prime}}\right)$ for $s>x$.

Proof. Take first $\mathbf{s}^{\prime}=\mathbf{s}$. We have that $r(\boldsymbol{\lambda})=x$ if and only if we have that $\boldsymbol{\lambda}$ is of the form

$$
\left(\lambda^{1}, \ldots, \lambda^{k}, \lambda^{1}, \ldots, \lambda^{k}, \ldots, \lambda^{1}, \ldots, \lambda^{k}\right)
$$

with $k=l / x$. Then Lemma 10 shows that this is equivalent to claim that $\boldsymbol{\lambda} \in \operatorname{Im}\left(\iota_{x}^{\mathbf{s}}\right)$ and the result follows.

Now, if $\mathbf{s}^{\prime} \in \mathbb{Z}^{l}$ in the same class as $\mathbf{s}=(0, e / l, \ldots,(l-1) e / l)$, then for all $\boldsymbol{\lambda} \in \Phi_{\mathbf{s}, e}(n)$, we have $D_{\mathbf{s}, e}^{\boldsymbol{\lambda}}=D_{\mathbf{s}^{\prime}, e}^{\Psi_{\mathbf{s} \rightarrow \mathbf{s}^{\prime}}^{e}(\boldsymbol{\lambda})}$ and one can conclude noticing that

$$
\boldsymbol{\lambda} \in \operatorname{Im}\left(\iota_{l / x}^{\mathbf{s}}\right) \Longleftrightarrow \Psi_{\mathbf{s}^{\prime} \rightarrow \mathbf{s}}^{e}(\boldsymbol{\lambda}) \in \operatorname{Im}\left(\iota_{l / x}^{\mathbf{s}^{\prime}}\right) .
$$

by the definition of the maps and the properties of crystal isomorphisms. 
Example 17. Take $l=4$ and $e=4$. Let $\mathbf{s}=(0,1,2,3)$. Then we have $\boldsymbol{\lambda}:=$ $(3.1,2,3.1,2) \in \Phi_{e, \mathbf{s}}(16)$. Then we have that $r(\boldsymbol{\lambda})=2$, this implies that $\operatorname{Res}\left(D_{\mathbf{s}, e}^{\boldsymbol{\lambda}}\right)$ splits in two simple $\mathcal{H}_{n}^{\prime}$-modules. Note that we have $\boldsymbol{\lambda}=\iota_{2}^{\mathbf{s}}(3.1,2)$. Set $\mathbf{s}=(0,13,26,39)$, this multicharge is very dominant and in the same orbit as $\mathbf{s}$. One can compute $\Psi_{\mathbf{s} \rightarrow \mathbf{s}^{\prime}}^{e}(\boldsymbol{\lambda})$ and we obtain $\boldsymbol{\lambda}^{\prime}=(2.1,1,3.2,2.1)$. So we have that $\operatorname{Res}\left(D_{\mathbf{s}^{\prime}, e}^{\boldsymbol{\lambda}^{\prime}}\right)$ splits in two simple $\mathcal{H}_{n}^{\prime}$-modules.

\subsection{The case $l=2$}

We assume in this part that $l=2$ and that $e$ is even. Then one can apply the results above. In particular, for all $\mathbf{s}$ in the orbit of $(0, e / 2)$ modulo $\widehat{\mathfrak{S}}_{2}$ and $\boldsymbol{\lambda} \in \Phi_{e, \mathbf{s}}(n)$, the $\mathcal{H}_{n}^{\prime}$-module $\operatorname{Res}\left(D_{\mathbf{s}, e}^{\boldsymbol{\lambda}}\right)$ splits into one or two simple modules.

The aim is to study the set of Uglov bipartitions $\boldsymbol{\lambda} \in \Phi_{e, \mathbf{s}}(n) \operatorname{such}$ that $\operatorname{Res}\left(D_{\mathbf{s}, e}^{\boldsymbol{\lambda}}\right) \operatorname{splits}$ into a sum of two simple modules. Such bipartitions will be called divided bipartitions for the multicharge $\mathbf{s}$. This notion strongly depends on $\mathbf{s}$. In the case where $\mathbf{s}=(0, e / 2)$, by the results above, these bipartitions correspond exactly to the bipartitions of the form $(\lambda, \lambda)$ in $\Phi_{e, \mathbf{s}}(n)$. This is exactly the set of bipartitions $(\lambda, \lambda)$ where $\lambda$ is an $e / 2$-regular partition of $n / 2$ by $\S 2.1$.

Proposition 18. Let $N \in \mathbb{Z}_{\geqslant 0}$. We have that $\left(\lambda^{1}, \lambda^{2}\right)$ is a divided bipartition for $\mathbf{s}=$ $(0, e / 2+N e)$ if and only if we have

$$
\Psi_{(0, e / 2+N e) \rightarrow(N e, e / 2)}^{e}\left(\lambda^{1}, \lambda^{2}\right)=\left(\lambda^{2}, \lambda^{1}\right)
$$

Proof. Let $\mathbf{s}=(0, e / 2+N e)$. Assume that $\left(\lambda^{1}, \lambda^{2}\right)$ is a divided bipartition and let $\left(i_{1}, \ldots, i_{n}\right) \in(\mathbb{Z} / e \mathbb{Z})^{n}$ be such that

$$
\widetilde{f}_{i_{1}}^{e, \mathbf{s}} \ldots \tilde{f}_{i_{n}}^{e, \mathbf{s}} \emptyset=\lambda
$$

Then we also have

$$
\tilde{f}_{i_{1}+e / 2}^{e, \mathbf{s}} \ldots \tilde{f}_{i_{n}+e / 2}^{e, \mathbf{s}} \emptyset=\boldsymbol{\lambda} .
$$

Now we have that $\mathbf{s}^{\prime}:=\tau . \mathbf{s}=(e / 2+N e, e)$ and by $\S 2.2$, we obtain:

$$
\widetilde{f}_{i_{1}}^{e, \mathbf{s}^{\prime}} \ldots{\widetilde{f_{i}}}_{i_{n}}^{e, \mathbf{s}^{\prime}} \emptyset=\left(\lambda^{2}, \lambda^{1}\right)
$$

Then we also have

$$
\widetilde{f}_{i_{1}+e / 2}^{e, \mathbf{s}^{\prime}} \ldots \widetilde{f}_{i_{n}+e / 2}^{e, \mathbf{s}^{\prime}} \emptyset=\left(\lambda^{2}, \lambda^{1}\right) .
$$

But now note that $\mathbf{s}=(N e, e / 2)=\mathbf{s}^{\prime}-(e / 2, e / 2)$ so it is clear that we obtain :

$$
\widetilde{f}_{i_{1}-e / 2}^{e,(N e, e / 2)} \ldots \widetilde{f}_{i_{n}-e / 2}^{e,(N e, e / 2)} \emptyset=\left(\lambda^{2}, \lambda^{1}\right),
$$

and this implies that $\Psi_{(0, e / 2+N e) \rightarrow(N e, e / 2)}^{e}\left(\lambda^{1}, \lambda^{2}\right)=\left(\lambda^{2}, \lambda^{1}\right)$. Reciprocally, assume that

$$
\Psi_{(0, e / 2+N e) \rightarrow(N e, e / 2)}^{e}\left(\lambda^{1}, \lambda^{2}\right)=\left(\lambda^{2}, \lambda^{1}\right) .
$$


Assume that $\left(i_{1}, \ldots, i_{n}\right) \in(\mathbb{Z} / e \mathbb{Z})^{n}$ is such that

$$
\widetilde{f}_{i_{1}}^{e, \mathbf{s}} \ldots \widetilde{f}_{i_{n}}^{e, \mathbf{s}} \emptyset=\left(\lambda^{1}, \lambda^{2}\right) .
$$

Then we have:

$$
\widetilde{f}_{i_{1}}^{e,(N e, e / 2)} \ldots \widetilde{f}_{i_{n}}^{e,(N e, e / 2)} \emptyset=\left(\lambda^{2}, \lambda^{1}\right) .
$$

We have $\tau$. $(N e, e / 2)=(e / 2, N e+e)$, and thus

$$
\widetilde{f}_{i_{1}}^{e,(e / 2, N e+e)} \ldots \widetilde{f}_{i_{n}}^{e,(e / 2, N e+e)} \emptyset=\left(\lambda^{1}, \lambda^{2}\right) .
$$

As $(0, e / 2+N e)=(e / 2, N e+e)-(e / 2, e / 2)$, we obtain

$$
\tilde{f}_{i_{1}+e / 2}^{e,(0, e / 2+N e)} \ldots \widetilde{f}_{i_{n}+e / 2}^{e,(0, e / 2+N e)} \emptyset=\left(\lambda^{1}, \lambda^{2}\right),
$$

which implies that $\left(\lambda^{1}, \lambda^{2}\right)$ is a divided partition (for $(0, e / 2+N e)$.

\section{References}

[1] S.Ariki. Representations of quantum algebras and combinatorics of Young tableaux. University Lecture Series, 26. American Mathematical Society, Providence, RI, 2002.

[2] M. Geck and N. Jacon. Representations of Hecke algebras at roots of unity. Algebra and Applications, 15. Springer-Verlag London, Ltd., London, 2011.

[3] G. Genet and N.Jacon. Modular representations of cyclotomic Hecke algebras of type $G(r, p, n)$. Int. Math. Res. Not. (2006), Article ID 93049.

[4] J. Hu. Crystal bases and simple modules for Hecke algebras of type $G(p, p, n)$. Represent.Theory, 11, (2007), 16-44.

[5] J. Hu. The number of simple modules for the Hecke algebras of type G(r,p,n). With an appendix by Xiaoyi Cui. J. Algebra 321 (2009), 3375-3396.

[6] H Lin and J. Hu. Crystal of affine $\widehat{\mathfrak{s l}}_{l}$ and Hecke algebras at a primitive $2 l$ root of unity. preprint arXiv:1905.07333.

[7] N. Jacon. Représentation modulaires d'algèbres de Hecke et d'algèbres de ArikiKoike. thèse de doctorat, Université Lyon I, 2004. available at https://tel. archives-ouvertes.fr/tel-00006383/fr/

[8] N. Jacon. On the one dimensional representations of Ariki-Koike algebras at roots of unity. J. Pure Appl. Algebra 221 (2017), 1298-1315.

[9] N. Jacon and C. Lecouvey. Crystal isomorphisms for irreducible highest weight $\mathcal{U}_{v}\left(\widehat{\mathfrak{s l}}_{e}\right)$-modules of higher level. Algebr. Represent. Theory 13, (2010), 467-489.

[10] S. Naito and D. Sagaki. Lakshmibai-Seshadri paths fixed by a diagram automorphism. J. Algebra, 245 (2001), 395-412. 